\title{
Short-Term Load Forecasting with Multi-Source Data Using Gated Recurrent Unit Neural Networks
}

\author{
Yixing Wang ${ }^{(\mathbb{D}}$, Meiqin Liu * ${ }^{(\mathbb{D}}$, Zhejing Bao and Senlin Zhang \\ College of Electrical Engineering, Zhejiang University, Hangzhou 310027, China; wangyixing@zju.edu.cn (Y.W.); \\ zjbao@zju.edu.cn (Z.B.); slzhang@zju.edu.cn (S.Z.) \\ * Correspondence: liumeiqin@zju.edu.cn; Tel.: +86-139-5800-7313
}

Received: 29 March 2018; Accepted: 1 May 2018; Published: 3 May 2018

check for updates

\begin{abstract}
Short-term load forecasting is an important task for the planning and reliable operation of power grids. High-accuracy forecasting for individual customers helps to make arrangements for generation and reduce electricity costs. Artificial intelligent methods have been applied to short-term load forecasting in past research, but most did not consider electricity use characteristics, efficiency, and more influential factors. In this paper, a method for short-term load forecasting with multi-source data using gated recurrent unit neural networks is proposed. The load data of customers are preprocessed by clustering to reduce the interference of electricity use characteristics. The environmental factors including date, weather and temperature are quantified to extend the input of the whole network so that multi-source information is considered. Gated recurrent unit neural networks are used for extracting temporal features with simpler architecture and less convergence time in the hidden layers. The detailed results of the real-world experiments are shown by the forecasting curve and mean absolute percentage error to prove the availability and superiority of the proposed method compared to the current forecasting methods.
\end{abstract}

Keywords: short-term load forecasting; artificial intelligence; gated recurrent unit; recurrent neural network; power grid

\section{Introduction}

Load forecasting is an essential part for energy management and distribution management in power grids. With the continuous development of the power grids and the increasing complexity of grid management, accurate load forecasting is a challenge [1,2]. High-accuracy power load forecasting for customers can make the reasonable arrangements of power generation to maintain the safety and stability of power supply and reduce electricity costs so that the economic and social benefit is improved. Moreover, forecasting at individual customer level can optimize power usage and help to balance the load and make detailed grid plans. Load forecasting is the process of estimating the future load value at a certain time with historical related data, which can be divided into long-term load forecasting, medium-term load forecasting and short-term load forecasting according to the forecasting time interval. Short-term load forecasting, which this paper focuses on, is the daily or weekly forecasting [3,4]. It is used for the daily or weekly schedule including generator unit control, load allocation and hydropower dispatching. With the increasing penetration of renewable energies, short-term load forecasting is fundamental for the reliability and economy of power systems.

Models of short-term load forecasting can be classified into two categories consisting of tradition statistic models and artificial intelligent models. Statistic models, such as regression analysis models and time sequence models, are researched and used frequently were previously limited by computing capability. Taylor et al. [5] proposed an autoregressive integrated moving average (ARIMA) model with an extension of Holt-Winters exponential smoothing for short-term load forecasting. Then, 
an power autoregressive conditional heteroskedasticity $(\mathrm{PARCH})$ method was presented for better performance [6]. These statistic models need fewer historical data and have a small amount of calculation. However, they require a higher stability of the original time sequences and do not consider the uncertain factors such as weather and holidays. Therefore, artificial intelligent models about forecasting, such as neural networks [7,8], fuzzy logic method [9] and support vector regression [10], were proposed with the development of computer science and smart grids. Recently, neural networks are becoming an active research topic in the area of artificial intelligence for its self-learning and fault tolerant ability. Some effective methodologies for load forecasting based on neural networks have been proposed in recent years. A neural network based method for the construction of prediction intervals was proposed by Quan et al. [7]. Lower upper bound estimation was applied and extended to develop prediction intervals using neural network models. The method resulted in higher quality for different types of prediction tasks. Ding [8] used separate predictive models based on neural networks for the daily average power and the day power variation forecasting in distribution systems. The prediction accuracy was improved with respect to naive models and time sequence models. The improvement of forecasting accuracy cannot be ignored, but, with the increasing complexity and scale of power grids, high-accuracy load forecasting with advanced network model and multi-source information is required.

Deep learning, proposed by Hinton [11,12], made a great impact on many research areas including fault diagnosis $[13,14]$ and load forecasting [15-17] by its strong learning ability. Recurrent neural network (RNNs), a deep learning framework, are good at dealing with temporal data because of its interconnected hidden units. It has proven successful in applications for speech recognition $[18,19]$, image captioning [20,21], and natural language processing [22,23]. Similarly, during the process of load forecasting, we need to mine and analyse large quantities of temporal data to make a prediction of time sequences. Therefore, RNNs are an effective method for load forecasting in power grids [24,25]. However, the vanishing gradient problem limits the performance of original RNNs. The later time nodes' perception of the previous ones decreases when RNNs become deep. To solve this problem, an improved network architecture called long short-term memory (LSTM) networks [26] were proposed, and have proven successful in dealing with time sequences for power grids faults [27,28]. Research on short-term load forecasting based on LSTM networks was put forward. Gensler et al. [29] showed the compared results for solar power forecasting about physical photovoltaic forecasting model, multi-layer perception, deep belief networks and auto-LSTM networks. It proved the LSTM networks with autoencoder had the lowest error. Zheng et al. [30] tackled the challenge of short-term load forecasting with proposing a novel scheme based on LSTM networks. The results showed that LSTM-based forecasting method can outperform traditional forecasting methods. Aiming at short-term load forecasting for both individual and aggregated residential loads, Kong et al. [31] proposed an LSTM recurrent neural network based framework with the input of day indices and holiday marks. Multiple benchmarks were tested in the real-world dataset and the proposed LSTM framework achieved the best performance. The research works mentioned above indicate the successful application of LSTM for load forecasting in power grids. However, load forecasting needs to be fast and accurate. The principle and structure of LSTM are complex with input gate, output gate, forget gate and cell, so the calculation is heavy for forecasting in a large scale grid. Gated recurrent unit (GRU) neural networks was proposed in 2014 [32], which combined the input gate and forget gate to a single gate called update gate. The model of a GRU is simpler compared with an LSTM block. It was proved on music datasets and ubisolf datasets that GRU's performance is better with less parameters about convergence time and required training epoches [33]. Lu et al. [34] proposed a multi-layer self-normalizing GRU model for short-term electricity load forecasting to overcome the exploding and vanishing gradient problem. However, short-term load forecasting for customers is influenced by factors including date, weather and temperature, which previous research did not consider seriously. People may need more energy when the day is cold or hot. Enterprises or factories may reduce their power consumption on holidays. 
In this paper, a method based on GRU neural networks with multi-source input data is proposed for short-term load forecasting in power grids. Moreover, this paper focuses on the load forecasting for individual customers, which is an important and tough problem because of the high volatility and uncertainty [30]. Therefore, before training the networks, we preprocess the customers' load data with clustering analysis to reduce the interference of the electricity use characteristics. Then, the customers are classified into three categories to form the training and test samples by K-means clustering algorithm. To obtain not only the load measurement data but also the important factors including date, weather and temperature, the input of the network are set as two parts. The temporal features of load measurement data are extracted by GRU neural networks. The merge layer is built to fuse the multi-source features. Then, we can get the forecasting results by training the whole network. The methodologies are described in detail in Section 2. The main contributions of this paper are as follows.

1. Trained samples are formed by clustering to reduce the interference of different characteristics of customers.

2. Multi-source data including date, weather and temperature are quantified for input so that the networks obtain more information for load forecasting.

3. The GRU units are introduced for more accurate and faster load forecasting of individual customers.

In general, the proposed method uses the clustering algorithm, quantified multi-source information and GRU neural network for short-term load forecasting, which past research did not consider comprehensively. The independent experiments in the paper verify the advantages of the proposed method. The rest of the paper is organized as follows. The methodology based on GRU Neural Networks for short-term load forecasting is proposed in Section 2. Then, the results and discussion of the simulation experiments are described to prove the availability and superiority of the proposed method in Section 3. Finally, the conclusion is made in Section 4.

\section{Methodology Based on GRU Neural Networks}

In this section, the methodology is proposed for short-term load forecasting with multi-source data using GRU Neural Networks. First, the basic model of GRU neural networks are introduced [32]. Then, data description and processing are elaborated. The load data are clustered by K-means clustering algorithm so that the load samples with similar characteristics in a few categories are obtained. This helps improve the performance of load forecasting for individual customers. In the last subsection, the whole proposed model based on GRU neural networks is shown in detail.

\subsection{Model of GRU Neural Networks}

Gated recurrent unit neural networks are the improvement framework based on RNNs. RNNs are improved artificial neural networks with the temporal input and output. Original neural networks only have connections between the units in different layers. However, in RNNs, there are connections between hidden units forming a directed cycle in the same layer. The network transmits the temporal information through these connections. Therefore, the RNNs outperform conventional neural networks in extracting the temporal features by these connections. A simple structure for an RNN is shown in Figure 1. The input and output are time sequences, which is different from original neural networks. The process of forward propagation is shown in Figure 1 and given by Equations (1)-(3).

$$
\begin{gathered}
a_{h}^{t}=\sum_{i=1}^{I} w_{i h} x_{i}^{t}+\sum_{h^{\prime}=1}^{H} w_{h^{\prime} h} s_{h^{\prime}}^{t-1} \\
s_{h}^{t}=f_{h}\left(a_{h}^{t}\right) \\
a_{o}^{t}=\sum_{h=1}^{H} w_{h o} s_{h}^{t}
\end{gathered}
$$


where $w$ is the weight; $a$ is the sum calculated through weights; $f$ is the activation function; $s$ is the value after calculation by the activation function; $t$ represents the current time of the network; $i$ is the number of input vectors; $h$ is the number of hidden vectors in $t$ is time; $h^{\prime}$ is the number of hidden vectors in $t-1$ time; and $o$ is the number of output vectors.

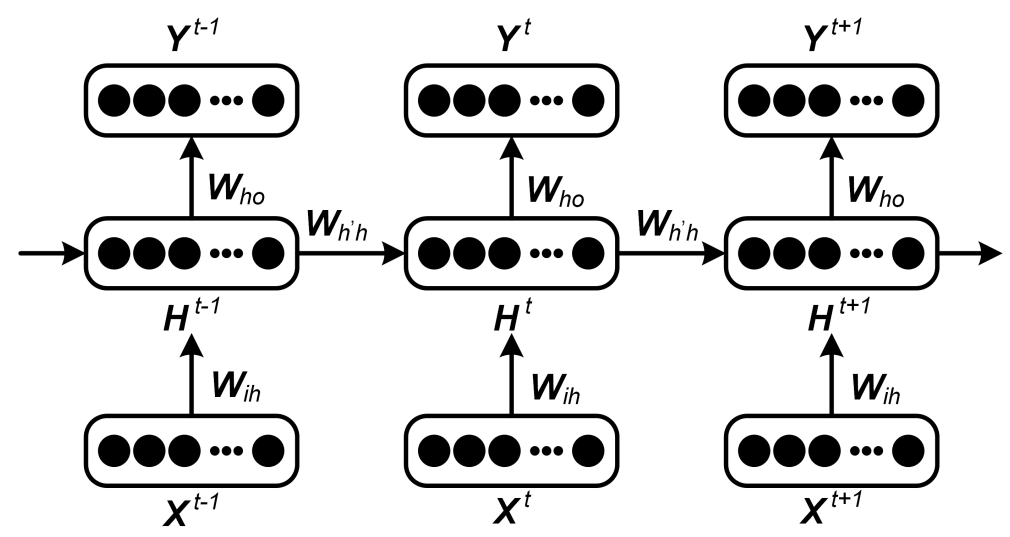

Figure 1. A simple RNN structure, where $X$ is the input unit, $\boldsymbol{H}$ is the hidden unit, $\boldsymbol{Y}$ is the output unit, and $W$ is the weight matrix.

Similar to conventional neural networks, RNNs can be trained by back-propagation through time [35] with the gradient descent method. As shown in Figure 1, each hidden layer unit receives not only the data input but also the output of the hidden layer in the last time step. The temporal information can be recorded and put into the calculation of the current output so that the dynamic changing process can be learned with this architecture. Therefore, RNNs are reasonable to predict the customer load curves in power grids. However, when the time sequence is longer, the information will reduce and disappear gradually through transferring in hidden units. The original RNNs have the vanishing gradient problem and the performance declines when dealing with long time sequences.

The vanishing gradient problem can be solved by adding control gates for remembering information in the process of data transfer. In LSTM networks, the hidden units of RNNs are replaced with LSTM blocks consisting of cell, input gate, output gate and forget gate. Moreover, the forget gate and input gate are combined into a single update gate in GRU neural network. The structure of GRU is shown in Figure 2.

The feedforward deduction process for GRU units is shown in Figure 2 and given by Equations (4)-(10).

$$
\begin{gathered}
a_{u}^{t}=\sum_{i=1}^{I} w_{i u} x_{i}^{t}+\sum_{h=1}^{H} w_{h u} s_{h}^{t-1} \\
s_{u}^{t}=f\left(a_{u}^{t}\right) \\
a_{r}^{t}=\sum_{i=1}^{I} w_{i r} x_{i}^{t}+\sum_{h=1}^{H} w_{h r} s_{h}^{t-1} \\
s_{r}^{t}=f\left(a_{r}^{t}\right) \\
\widetilde{a}_{h^{\prime}}^{t}=s_{u}^{t} \sum_{h=1}^{H} w_{h h^{\prime}} s_{h}^{t-1}+\sum_{i=1}^{I} w_{i h^{\prime}} x_{i}^{t} \\
\widetilde{s}_{h^{\prime}}^{t}=\phi\left(\widetilde{a}_{h^{\prime}}^{t}\right) \\
s_{h}^{t}=\left(1-s_{u}^{t}\right) \widetilde{s}_{h^{\prime}}^{t}+s_{u}^{t} s_{h}^{t-1}
\end{gathered}
$$




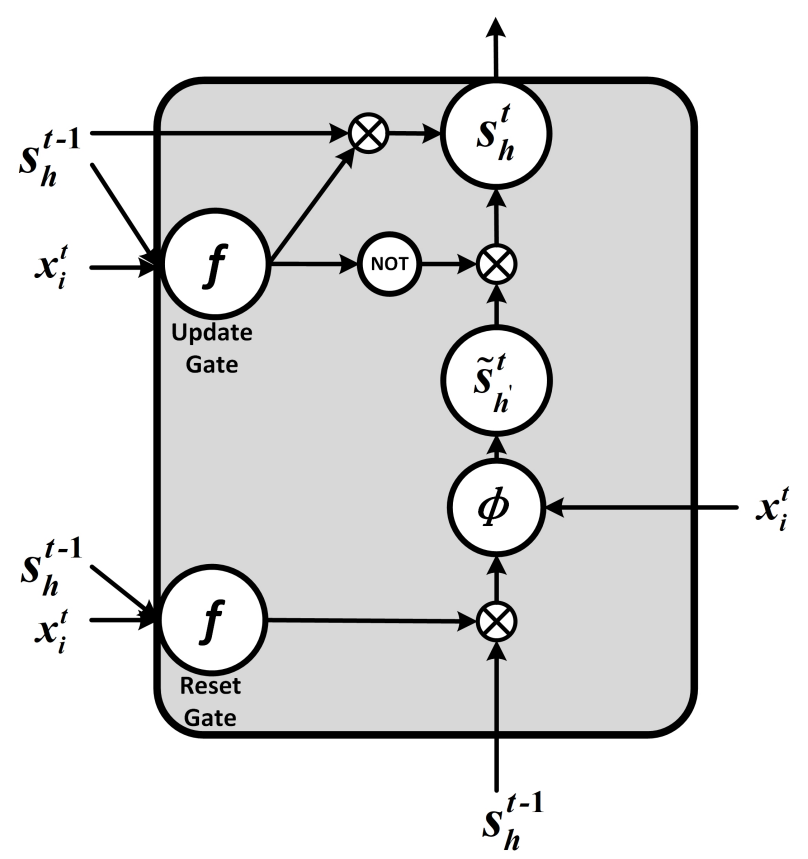

Figure 2. Inner structure of GRU, where all arrows represent the weights between gates and units and the units of $f$ and $\phi$ are the activation functions. The parameters are explained in detail after the Equations (4)-(10).

where $u$ is the number of update gate vector; $r$ is the number of reset gate vector; $h$ is the number of hidden vectors at $t$ time step; $h^{\prime}$ is the number of hidden vectors at $t-1$ time step; $f$ and $\phi$ are the activation functions; $f$ is the sigmoid function and $\phi$ is the tanh function generally; and $\widetilde{s}_{h^{\prime}}^{t}$ means the new memory of hidden units at $t$ time step.

According to Figure 2, the new memory $\widetilde{s}_{h^{\prime}}^{t}$ is generated by the input $x_{i}^{t}$ at the current time step and the hidden unit state $s_{h}^{t-1}$ at the last time step, which means the new memory can combine the new information and the historical information. The reset gate determines the importance of $s_{h}^{t-1}$ to $\widetilde{s}_{h^{\prime}}^{t}$. If the historical information $s_{h}^{t-1}$ is not related to new memory, the reset gate can completely eliminate the information in the past. The update gate determines the degree of transfer from $s_{h}^{t-1}$ to $s_{h}^{t}$. If $s_{u}^{t} \approx 1, s_{h}^{t-1}$ is almost completely passed to $s_{h}^{t}$. If $s_{u}^{t} \approx 0, \widetilde{s}_{h^{\prime}}^{t}$ is passed to $s_{h}^{t}$. The structure shown in Figure 2 results in a long memory in GRU neural networks. The memory mechanism solves the vanishing gradient problem of original RNNs. Moreover, compared to LSTM networks, GRU neural networks merge the input gate and forget gate, and fuse the cell units and hidden units in LSTM block. It maintains the performance with simpler architecture, less parameters and less convergence time [33]. Correspondingly, GRU neural networks are trained by back-propagation through time as RNNs [35].

\subsection{Data Description}

The real-world load data of individual customers in Wanjiang area is recorded from Dongguan Power Supply Bureau of China Southern Power Grid in Guangdong Province, China during 2012-2014. The topology structure of Wanjiang area is shown in Figure 3. There are 36 feeders connecting to the load sides in the Wanjiang area, i.e., Feeders $1-36$. The active power is extracted for load forecasting from these feeders. The sampling period is $15 \mathrm{~min}$ as the meter record data. The load curve of a customer, No. 53990001, from Feeder 2 during a month is shown in Figure 4, where the different load characteristics of the customer on each day can be concluded. 


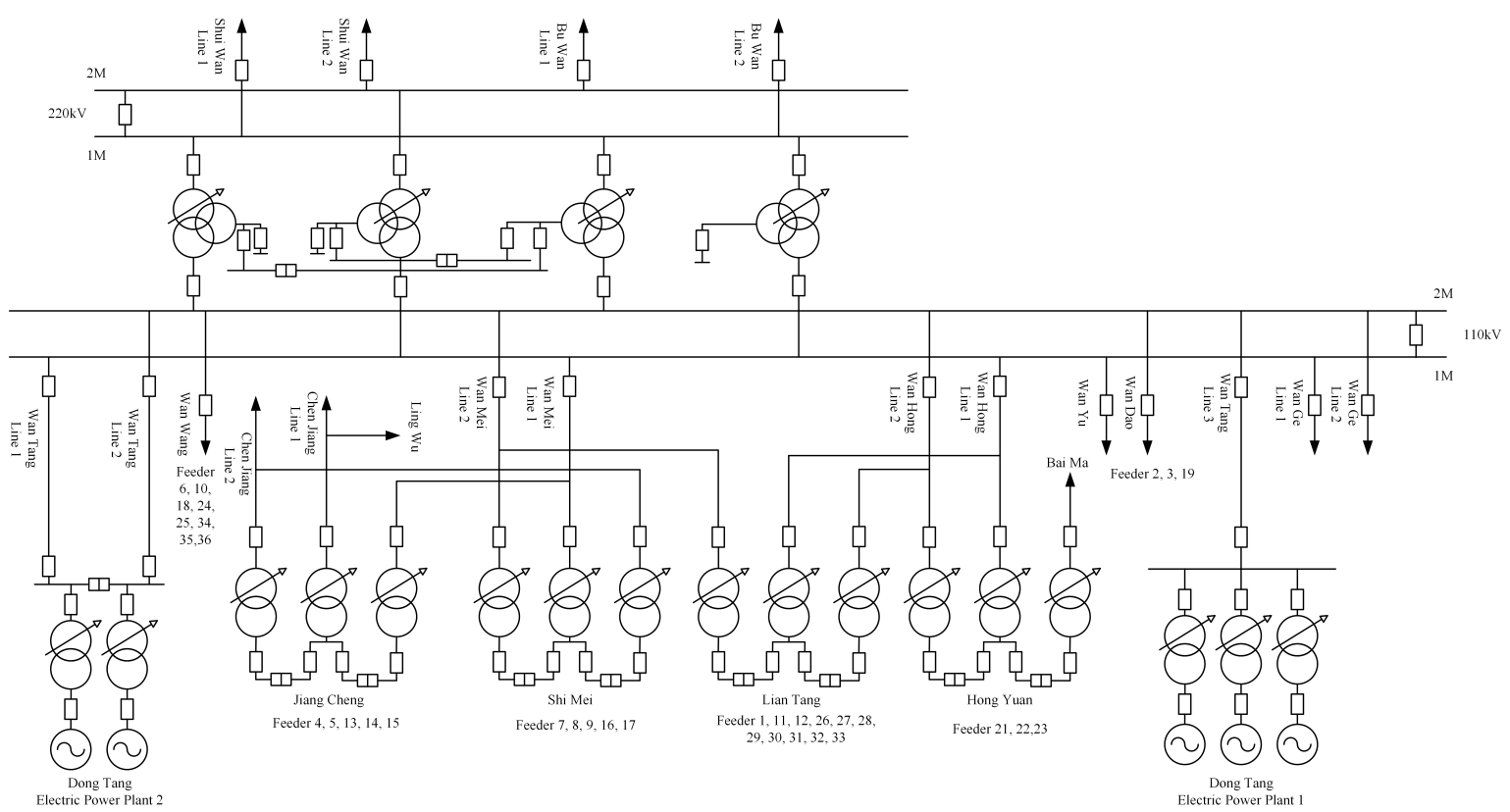

Figure 3. Primary electrical system in Wanjiang area above $110 \mathrm{kv}$, including electric power plants, transmission buses, converting stations, and user loads. The feeders are marked under their corresponding load sides.

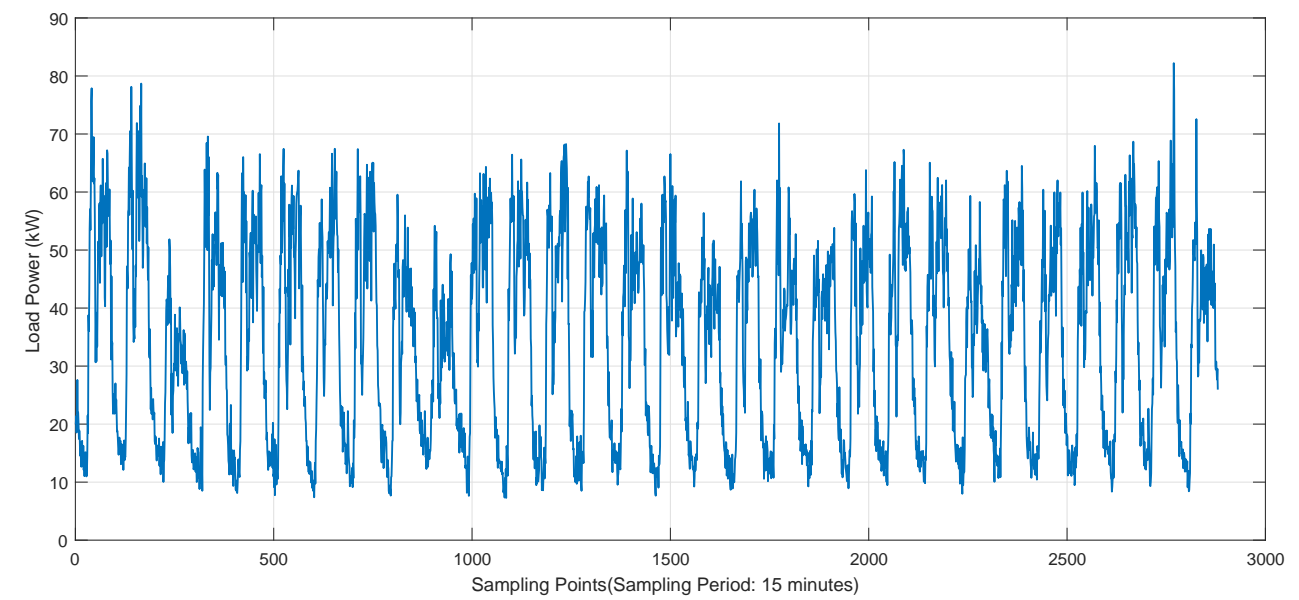

Figure 4. Load curve of Customer 53990001 from Feeder 2 during a month, where the sampling period is $15 \mathrm{~min}$.

Besides the historical load curves, short-term load forecasting is influenced by the factors of date, weather and temperature. The real historical data of weather and temperature in the corresponding area in Dongguan City were obtained online from the weather forecast websites. The categories of weather include sunny, cloud, overcast, light rain, shower, heavy rain, typhoon and snow. The date features can be found in calendars.

\subsection{Clustering and Quantization}

The custom of electricity use and characteristics of load curve are different among the different categories of customers such as industrial customers, residential customers and institution customers. The different characteristics would affect the performance of forecasting. Training forecasting networks with each customer separately would be a huge computation and storage problem. Therefore, in 
the proposed method, the load curve samples are divided into certain categories using K-means clustering algorithm. Samples with similar characteristics form a certain category, which form the input of GRU neural networks for the corresponding customers. K-means clustering algorithm is a simple and available method for clustering through unsupervised learning with fast convergence and less parameters. The only parameter, $K$, number of clustering category, can be determined by Elbow method with the turning point of loss function curve.

Suppose the input sample is $S=x_{1}, x_{2}, \ldots, x_{m}$. The algorithm is shown as follows.

1. Randomly initialize $K$ clustering centroids $c_{1}, c_{2}, \ldots, c_{K}$.

2. For $i=1,2, \ldots, m$, label each sample $\boldsymbol{x}_{i}$ with the clustering centroid closest to $\boldsymbol{x}_{i}$, getting $K$ categories noted by $G_{k}$.

$$
\text { label }_{i}=\underset{1 \leq k \leq K}{\arg \min }\left\|\boldsymbol{x}_{i}-\boldsymbol{c}_{k}\right\|, i=1,2, \ldots, m
$$

3. For $k=1,2, \ldots, K$, average the samples assigned to $G_{k}$ to update $c_{k}$.

$$
c_{k}=\frac{1}{\left|G_{k}\right|} \sum_{i \in G_{k}} x_{i}, k=1,2, \ldots, K
$$

4. Repeat Steps 2 and 3 until the change of clustering centroid or the loss function of clustering less than a set threshold. The loss function is given by Equation (13), where $x_{j}$ is the samples in categories $G_{k}, j=1,2, \ldots, n_{k}$ and $n_{k}$ is the number of samples in categories $G_{k}$.

$$
J\left(c_{1}, c_{2}, \ldots, c_{K}\right)=\frac{1}{2} \sum_{k=1}^{K} \sum_{j=1}^{n_{k}}\left\|x_{j}-c_{k}\right\|
$$

Moreover, the factors of date, weather and temperature should be added into input with quantization. First, the power consumption should be different between weekdays and weekends. The official holidays are also an important factor, so we quantify the date index as shown in Table 1 , where the index of official holidays is 1 no matter what day it is. Similarly, the weather and temperature are quantified according to their inner relations, as shown in Tables 2 and 3.

Table 1. Quantization for the factors of date.

\begin{tabular}{ccccccccc}
\hline Date (D) & Mon. & Tues. & Wed. & Thur. & Fri. & Sat. & Sun. & Official Holidays \\
\hline Index & 0 & 0.02 & 0.04 & 0.06 & 0.08 & 0.6 & 0.8 & 1 \\
\hline
\end{tabular}

Table 2. Quantization for the factors of weather.

\begin{tabular}{ccccccccc}
\hline Weather $(\mathbf{W})$ & Sunny & Cloud & Overcast & Light Rain & Shower & Heavy Rain & Typhoon & Snow \\
\hline Index & 0 & 0.1 & 0.2 & 0.4 & 0.5 & 0.6 & 0.8 & 1 \\
\hline
\end{tabular}

Table 3. Quantization for the factors of temperature.

\begin{tabular}{ccccccc}
\hline Temperature $\left(\mathbf{T} /{ }^{\circ} \mathbf{C}\right)$ & $\mathbf{T} \leq \mathbf{0}$ & $\mathbf{0}<\mathbf{T} \leq \mathbf{1 0}$ & $\mathbf{1 0}<\mathbf{T} \leq \mathbf{2 0}$ & $\mathbf{2 0}<\mathbf{T} \leq \mathbf{3 0}$ & $\mathbf{3 0}<\mathbf{T} \leq \mathbf{4 0}$ & $\mathbf{T} \geq \mathbf{4 0}$ \\
\hline Index & 0 & 0.2 & 0.4 & 0.6 & 0.8 & 1 \\
\hline
\end{tabular}

\subsection{The Proposed Framework Based on GRU Neural Networks}

The schematic diagram of proposed framework based on GRU neural networks for short-term load forecasting is shown in Figure 5. The individual customers are clustered into a few categories for more accurate forecasting. The samples are recorded from the categories where the customer to 
be predicted locates in. The load measurement data of individual customers in one day is extracted as a sample for short-term load forecasting, noted by $\boldsymbol{P}$. The dimension of $\boldsymbol{P}$ is 96 with the $15 \mathrm{~min}$ sampling period. Then, the samples are reshaped into two-dimension for the input of GRU neural networks. Considering the influencing factors date $D$, weather $W$ and temperature $T$, date $D_{\mathrm{p}}$, weather $W_{\mathrm{p}}$ and temperature $T_{\mathrm{p}}$ on the forecasting day are added to the another input of the GRU neural networks. Considering the general factor of date, the prediction interval is set to seven days. Therefore, the load measurement data $\boldsymbol{P}_{1}$ on the day in the last week from the forecasting day, $D_{p}, W_{p}$ and $T_{p}$, are recorded as the overall input. The load measurement data $\boldsymbol{P}_{\mathrm{p}}$ on the forecasting day are recorded as the output, whose dimension is 96 . Therefore, the input $X$ and output $Y$ of samples are given by Equations (14) and (15).

$$
\begin{gathered}
\boldsymbol{X}=\left\{\boldsymbol{P}_{1} ; D_{\mathrm{p}}, W_{\mathrm{p}}, T_{\mathrm{p}}\right\} \\
\boldsymbol{Y}=\left\{\boldsymbol{P}_{\mathrm{p}}\right\}
\end{gathered}
$$

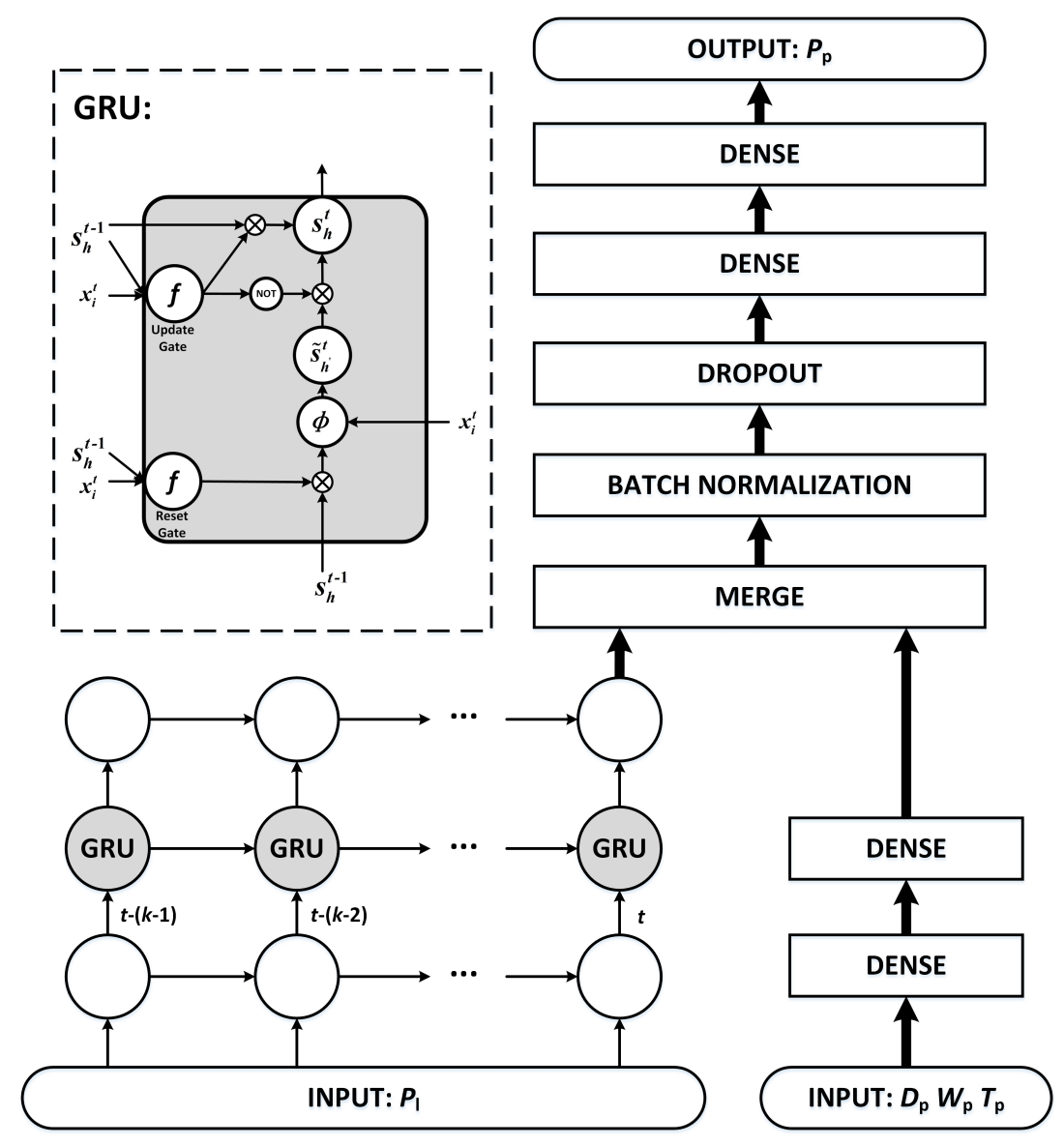

Figure 5. Schematic diagram of proposed framework based on GRU Neural Networks for short-term load forecasting, where $k$ is the number of hidden units and $t$ is the time step. The parameters of GRU units are clarified in Section 2.1. The input and output parameters are explained in the next subsection.

The features from GRU neural networks and fully connected neural network are merged with the concatenating mode and passes through batch normalization and dropout layer to avoid overfitting and increase the learning efficiency. The principle is that batch normalization can avoid the gradient vanishing of falling into the saturated zone, and that the better performance in fixed combination is avoided when random neurons do not work in a dropout layer. Then, two-layer fully connected neural network are added before the output for learning and generalization ability. With training by back-propagation through time, the whole network implements the short-term load forecasting 
for individual customers. The structure can be extended if there is more information in the practical situation. The basic theory is also acceptable for medium-term load forecasting and long-term load forecasting, but different influence factors should be considered and the model should be changed with different input, output, and inner structure for good performance.

\section{Experiments and Results}

In this section, the experiments are described in detail and the results are shown in figures and tables. The specific discussion for results is elaborated after the results and prove the improved performance compared to other methods. The data for experiments are recorded in Section 2.2.

\subsection{Clustering Analysis for Load Curve of Individual Customers}

Before the short-term load forecasting using GRU neural networks, the load curves of individual customers are clustered to different categories for samples with K-means clustering algorithm. The parameter $K$ is selected as 3 by Elbow method. There are 746 customers in the Wanjiang area in Dongguan city. The load measurement data should be processed with $0-1$ standardization to the same scale for clustering to reduce the impact of different magnitudes and dimensions. The clustering is done for 10 times with load curves in 10 days for the individual customers. The clustering results are obtained with the average results in 10 days and the number of each clustering category is shown in Table 4 . The standardized curves for 30 selected customers in three categories on a weekday are shown in Figures 6-8.

Table 4. Number of each clustering category.

\begin{tabular}{cccc}
\hline Categories & Category $\mathbf{1}$ & Category 2 & Category $\mathbf{3}$ \\
\hline Number of Customers & 221 & 308 & 217 \\
\hline
\end{tabular}

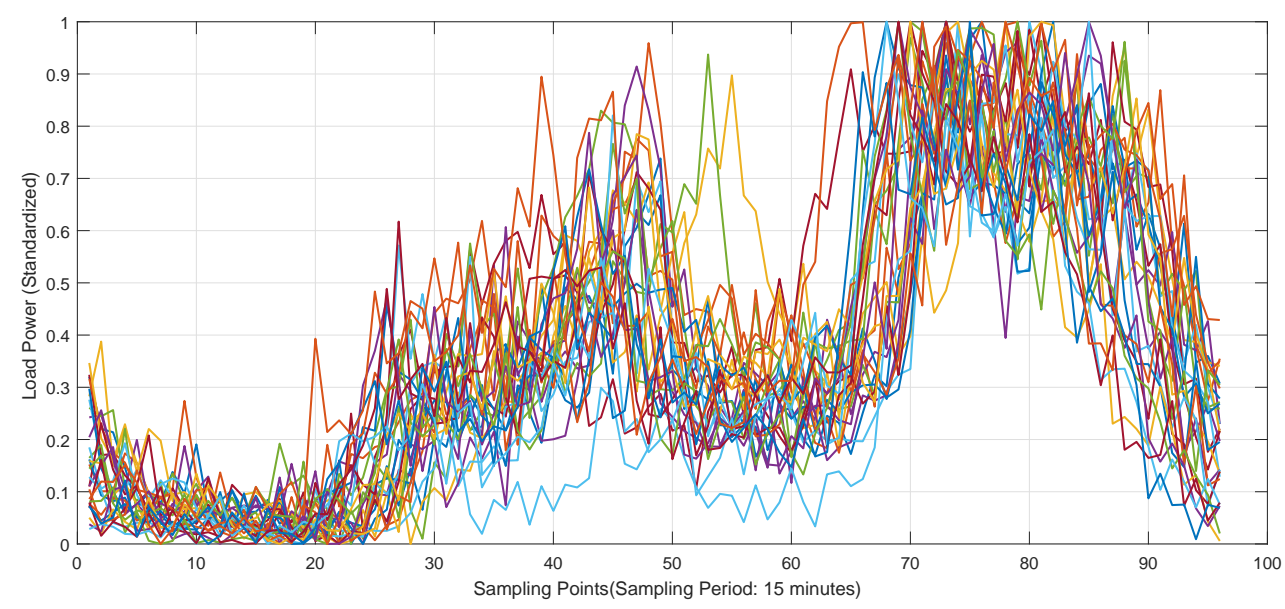

Figure 6. Load curves of 30 customers in Category 1.

As can be seen in Figures 6-8, different customers have different characteristics of electricity use. According to Figure 6, there are two electric peaks in a day. The evening peak is higher than the noon peak. The classic representation of this characteristic in Figure 6 is residential customers. Different from Figure 6, Figure 7 maintains the peak from 9 a.m. to late at night except noon. They are the general load curves of industry and business customers. In Figure 8, there are two electric peaks in the morning and afternoon. It should belong to the government and institutional customers. Even though a few customers have differences with the overall curve, this is the best clustering for them and it does not influence the overall performance greatly. With the clustering of individual customers, the 
networks can be trained with samples in the same category according to the customer to be predicted, so that the interference of electricity use characteristics can be reduced.

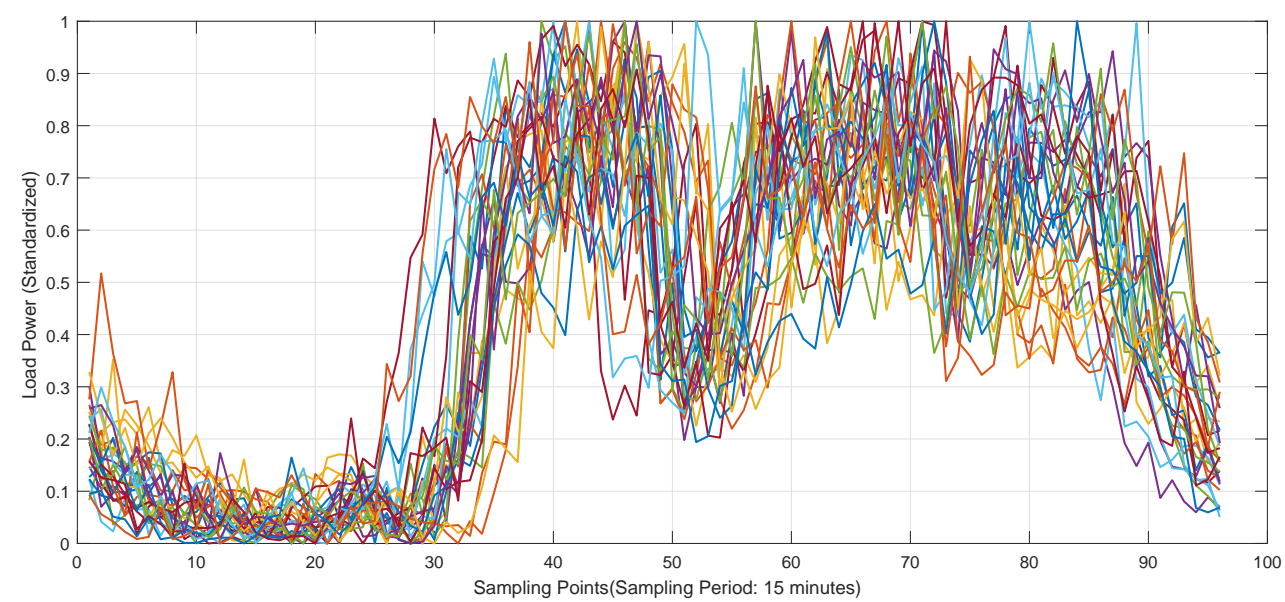

Figure 7. Load curves of 30 customers in Category 2.

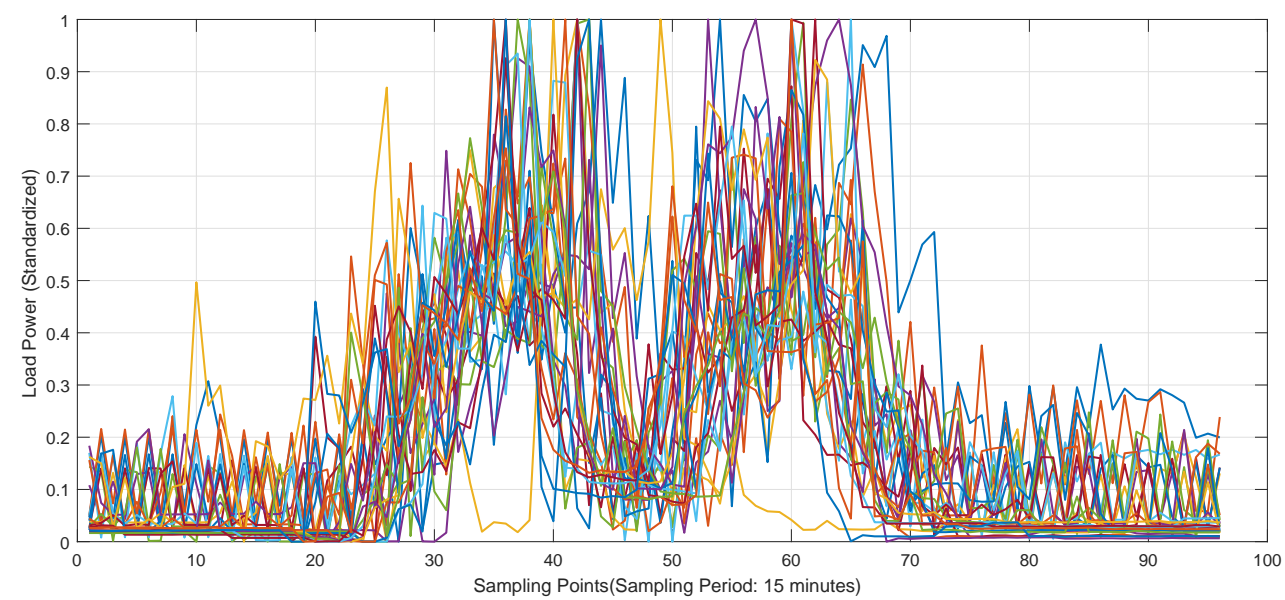

Figure 8. Load curves of 30 customers in Category 3.

\subsection{The Detailed Network Structure and Parameters}

The detailed structure of whole network are shown in Table 5. The parameters of the network are set as shown in Table 6. The structure and parameters are set for better performance according to the multiple experiments for customers in Wanjiang area. The "RMSprop" optimizer is chosen for its better performance in recurrent neural networks. The parameters can be adjusted for the different practical situations. In this paper, the number of epoch is set to 200 for the proposed method and can be adjusted for the compared methods. The training is stopped when the error decreases to a steady state. 
Table 5. Number of units in the proposed network.

\begin{tabular}{cccc}
\hline Layer & Number of Units & Layer & Number of Units \\
\hline INPUT: $P_{1}$ & $(6,16)$ & INPUT: $D_{p} W_{p} T_{p}$ & 3 \\
BATCH NORMALIZATION & $(6,16)$ & DENSE1 & 100 \\
GRU & 300 & DENSE2 & 100 \\
MERGE & 400 & & \\
DROPOUT & 400 & & \\
DENSE3 & 100 & & \\
DENSE4 & 100 & & \\
OUTPUT: $P_{\mathrm{p}}$ & 96 & & \\
\hline
\end{tabular}

Table 6. Parameter setting in the proposed network.

\begin{tabular}{cc}
\hline Parameters & Value \\
\hline Input Time Steps & 6 \\
Input Dimension & 16 \\
Batch Size & 30 \\
Epoch & 200 \\
Optimizer & RMSprop \\
Learning Rate & 0.001 \\
Decay & 0.9 \\
Dropout Rate & 0.6 \\
\hline
\end{tabular}

\subsection{Comparison of Results of Proposed Method}

The results of the proposed method are shown as follows. In the experiments, the training samples are recorded from the load data in the period from October 2012 to September 2013 while the test samples are recorded from load data in the period from October to December of 2013. The number of recorded training samples and test samples of each categories is 36,000 and 9000, respectively, with 100 customers in a category. The ratio of sample number is 4:1. Mean absolute percentage error (MAPE) is the classic evaluation index for load forecasting. The computational formula is given by Equations (16) and (17), where $n=96$ represents the dimension of samples and $m$ represents the number of test samples.

$$
\begin{gathered}
\text { MAPE }=\frac{\sum_{j=1}^{m}\left|E_{j}\right|}{m} \times 100 \% \\
E_{j}=\frac{\sum_{i=1}^{n}\left|P_{\mathrm{p}, i j}-P_{1, i j}\right|}{n}
\end{gathered}
$$

Customer 53990001 is selected from Category 2 for the forecasting customer. The MAPEs during a training period for Category 2 are shown in Figure 9 when the parameters are set as shown in Table 6 . The compared curves of actual load and forecasting load using the proposed method on 18 November for Customer 53990001 are shown in Figure 10. The MAPE for Customer 53990001 on 18 November is $10.23 \%$. The compared curves of actual load and forecasting load from 18 to 24 November for Customer 53990001 are shown in Figure 11. The MAPE for Customer 53990001 in this week is 10.97\%. In Figures 10 and 11, the error in sample points of one day is basically average and becomes larger when the curve comes to a peak. It is reasonable because the high or low peak is not reachable in most cases. The network should balance the prediction results for most situations during the training process. According to Figure 9, the MAPE decreases to a steady state as the epoch increases to 200. According to Figures 10 and 11, the forecasting curve is close to the actual curve, which proves the availability of the proposed method. 


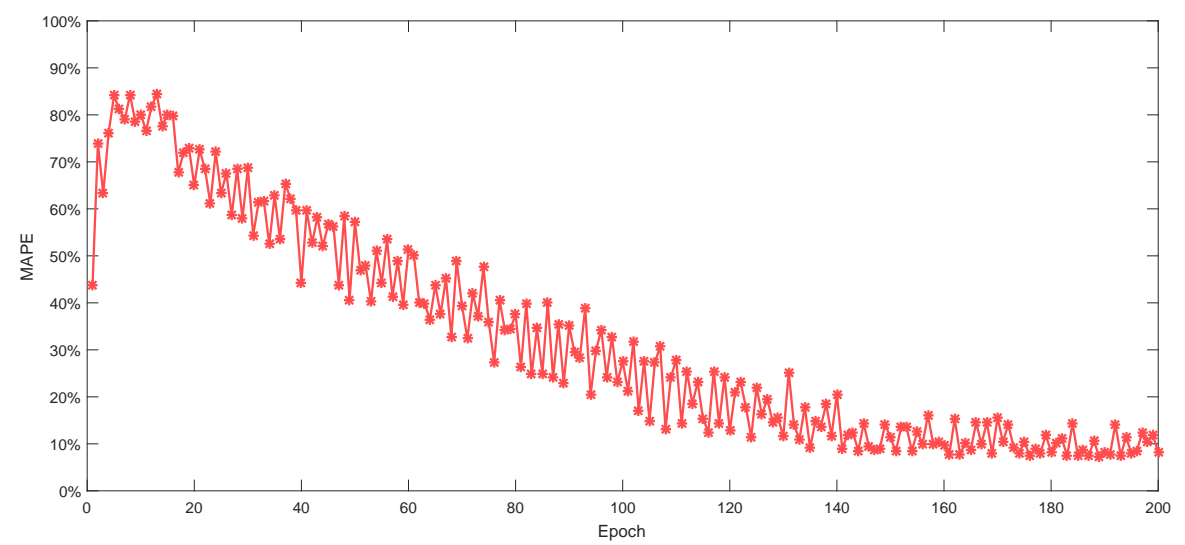

Figure 9. MAPEs during a training period for the Category 2.

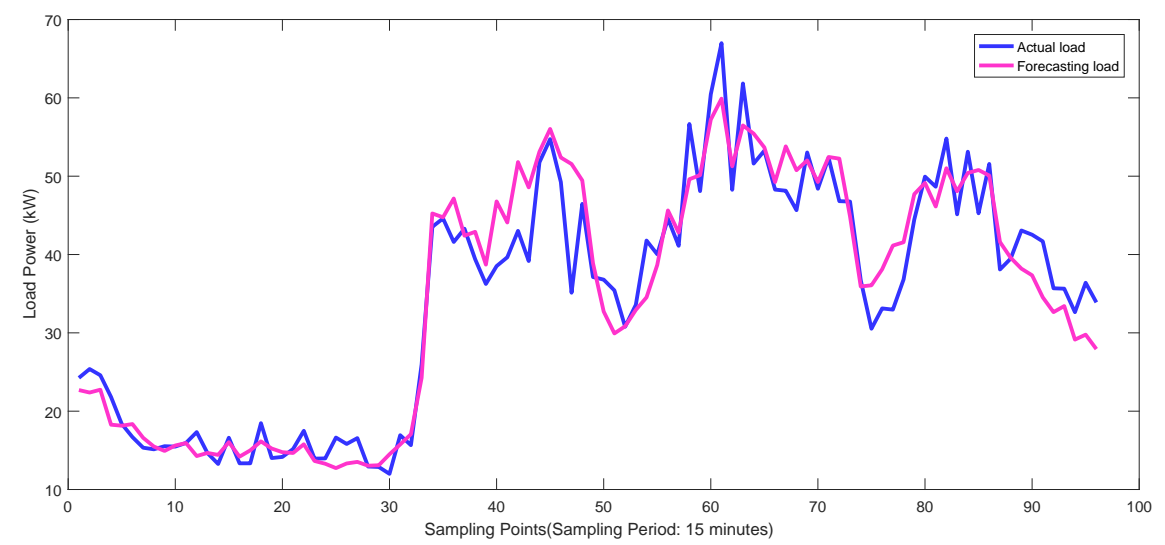

Figure 10. Compared curves of actual load and forecasting load in a day for Customer 53990001.

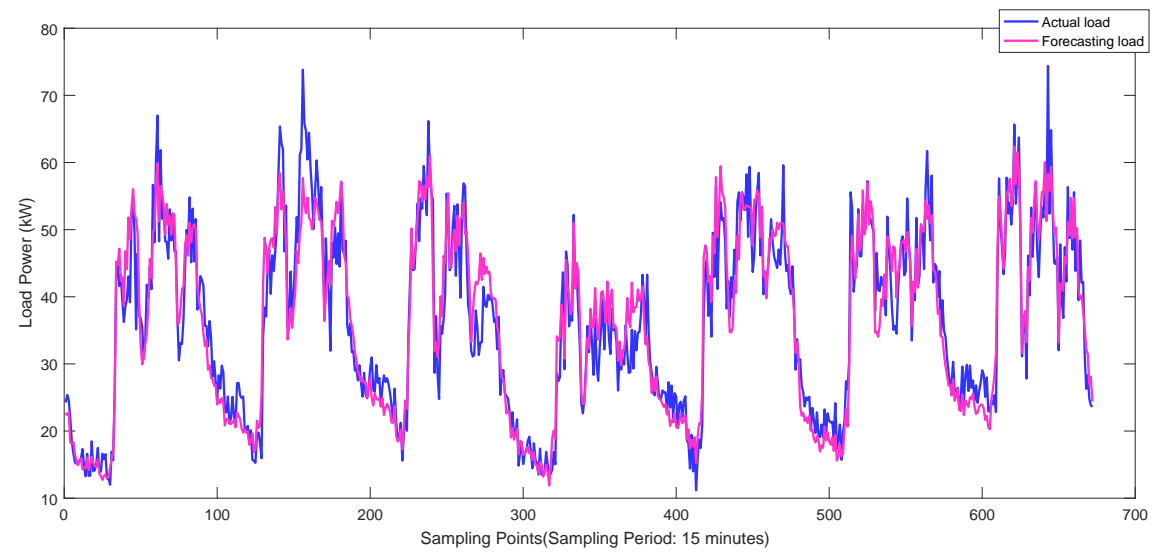

Figure 11. Compared curves of actual load and forecasting load in a week for Customer 53990001.

The samples are preprocessed by K-means clustering algorithm to form three categories for training. We performed a comparative experiment with variable-controlling approach about clustering. The compared results of Customer 53990001 on four different days of November 2013 are shown in Figure 12. The compared MAPEs of prediction on 18 November for nine customers in three categories from different feeders are shown in Table 7. It can be concluded that the forecasting curve without clustering deviates from the actual curve and that its MAPE is larger. The reason is that different 
characteristics of electricity use create a bad effect for short-term load forecasting. The effect reduces when we use corresponding trained networks for different customers. Therefore, the performance is generally improved by clustering.

Table 7. Compared MAPEs for nine customers in three categories with or without clustering.

\begin{tabular}{ccccc}
\hline Customer & Category & Feeder & MAPE with Clustering & MAPE without Clustering \\
\hline 37148000 & 1 & 2 & $10.80 \%$ & $22.98 \%$ \\
51690000 & 1 & 7 & $9.25 \%$ & $21.17 \%$ \\
37165000 & 1 & 7 & $11.12 \%$ & $20.12 \%$ \\
53990001 & 2 & 2 & $10.07 \%$ & $27.31 \%$ \\
54265001 & 2 & 3 & $11.91 \%$ & $22.72 \%$ \\
54265002 & 2 & 3 & $10.76 \%$ & $28.45 \%$ \\
31624001 & 3 & 35 & $13.56 \%$ & $21.12 \%$ \\
41661001 & 3 & 34 & $12.23 \%$ & $24.85 \%$ \\
76242001 & 3 & 33 & $9.98 \%$ & $28.38 \%$ \\
\hline
\end{tabular}
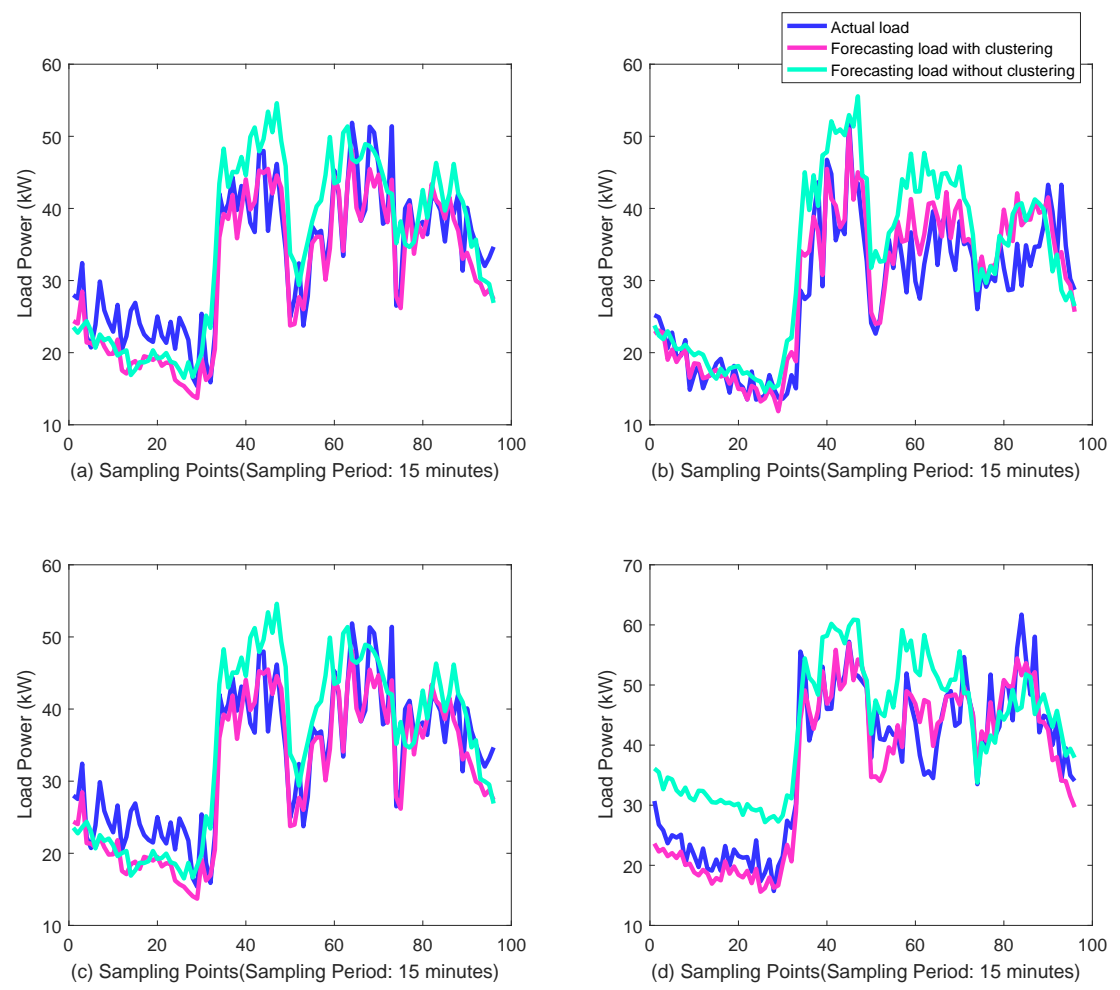

Figure 12. Compared curves of actual load and forecasting load of Customer 53990001 with or without clustering: (a-d) the results for four different days in November 2013.

The input of proposed network includes $D_{\mathrm{p}}, W_{\mathrm{p}}, T_{\mathrm{p}}$ and $\boldsymbol{P}_{1}$, which means that the network obtains and fuses the previous load changing process and other environmental information. In this case, we removed the input layer and the following fully connected layers in the network. The comparison results of Customer 53990001 with multi-source or only load data input are shown in Figure 13. The compared MAPEs for nine customers in three categories from different feeders are shown in Table 8. The experimental condition is the same as the one above. It can be concluded that the performance of only using load data is obviously poorer. Although the change shape is similar to actual, the curves deviate from the actual curves. Correspondingly, the MAPEs are larger. The reason is that date, weather and temperature are necessary factors to consider during short-term load forecasting processing. People would raise their load on a hot or cold day, even a rainy or snowy day. 
Resident customers may increase electricity consumption on weekends but business customers may not. These are some obvious reasons why we should consider the environment factors.

Table 8. Compared MAPEs for nine customers in three categories with multi-source data or only load data.

\begin{tabular}{ccccc}
\hline Customer & Category & Feeder & MAPE with Multi-Source Data & MAPE with only Load Data \\
\hline 37148000 & 1 & 2 & $10.80 \%$ & $15.09 \%$ \\
51690000 & 1 & 7 & $9.25 \%$ & $15.06 \%$ \\
37165000 & 1 & 7 & $11.12 \%$ & $16.50 \%$ \\
53990001 & 2 & 2 & $10.07 \%$ & $18.56 \%$ \\
54265001 & 2 & 3 & $11.91 \%$ & $17.36 \%$ \\
54265002 & 2 & 3 & $10.76 \%$ & $15.99 \%$ \\
31624001 & 3 & 35 & $13.56 \%$ & $15.89 \%$ \\
41661001 & 3 & 34 & $12.23 \%$ & $16.46 \%$ \\
76242001 & 3 & 33 & $9.98 \%$ & $17.33 \%$ \\
\hline
\end{tabular}
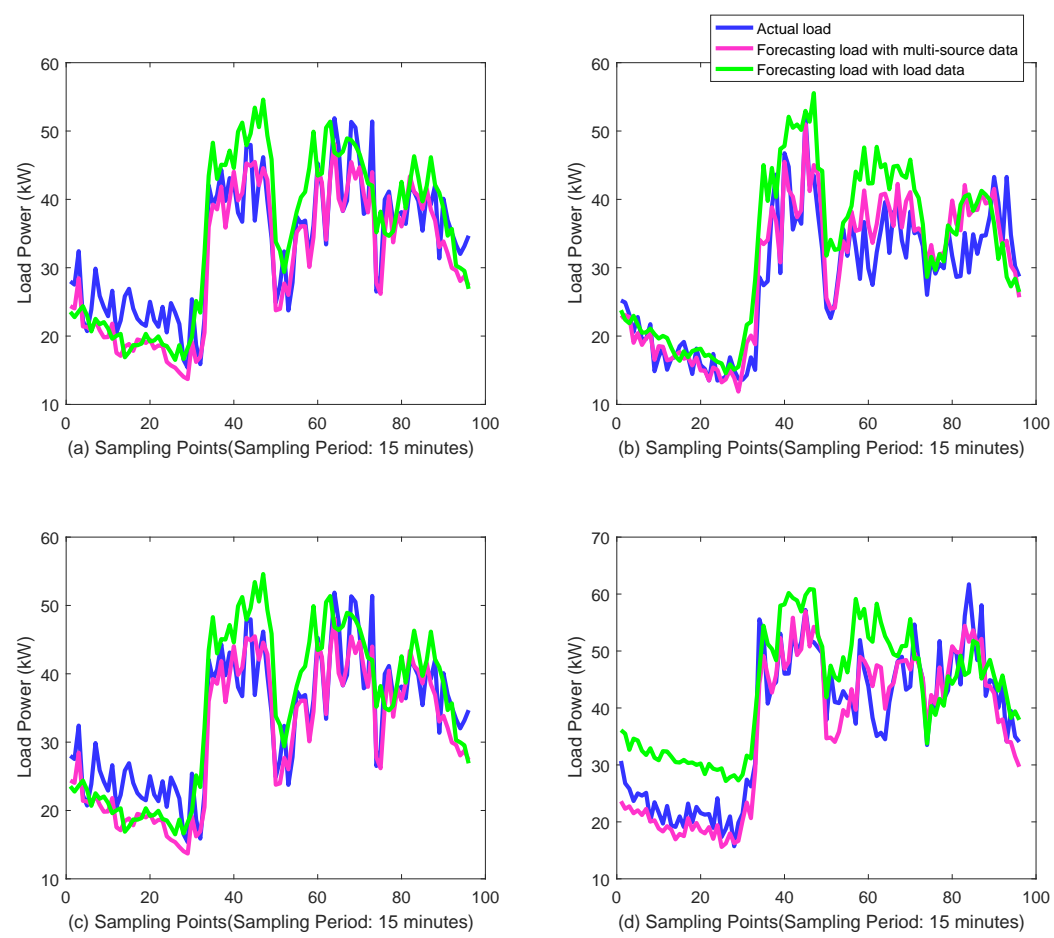

Figure 13. Comparison curve of actual load and forecasting load of Customer 53990001 with or without multi-source data: (a-d) the results for the same four days in November 2013 as the experiment in Figure 12.

It can be concluded from the two experiments that the MAPEs are floating in a certain degree. The maximal MAPEs of all samples in the conditions of the two experiments are shown in Table 9. The maximal MAPE without clustering and with only load data is significantly larger than the proposed method with clustering and multi-source data. The maximal MAPE of proposed method is $15.12 \%$, which is acceptable for load forecasting of individual customers.

Table 9. Maximal MAPEs in different conditions.

\begin{tabular}{cccc}
\hline Conditions & Forecast without Clustering & Forecast with only Load Data & Proposed Method \\
\hline Maximal MAPE & $30.25 \%$ & $21.87 \%$ & $15.12 \%$ \\
\hline
\end{tabular}


The performances are good with LSTM networks in dealing with time sequence but there are more parameters to train compared with GRU neural networks. In the proposed network, the GRU layers have 285,300 parameters to train while the LSTM layers have 380,400 parameters with the same architecture. The cost time for training with LSTM network is about $20 \%$ longer than training with GRU neural networks in the experiments in this paper. The MAPEs of network with LSTM and GRU layer in the same architecture with the same samples in Category 2 during the training process are shown in Figure 14. We can conclude that GRU neural networks do better in both convergence speed and training time, which depends on the improved single structure of GRU units.

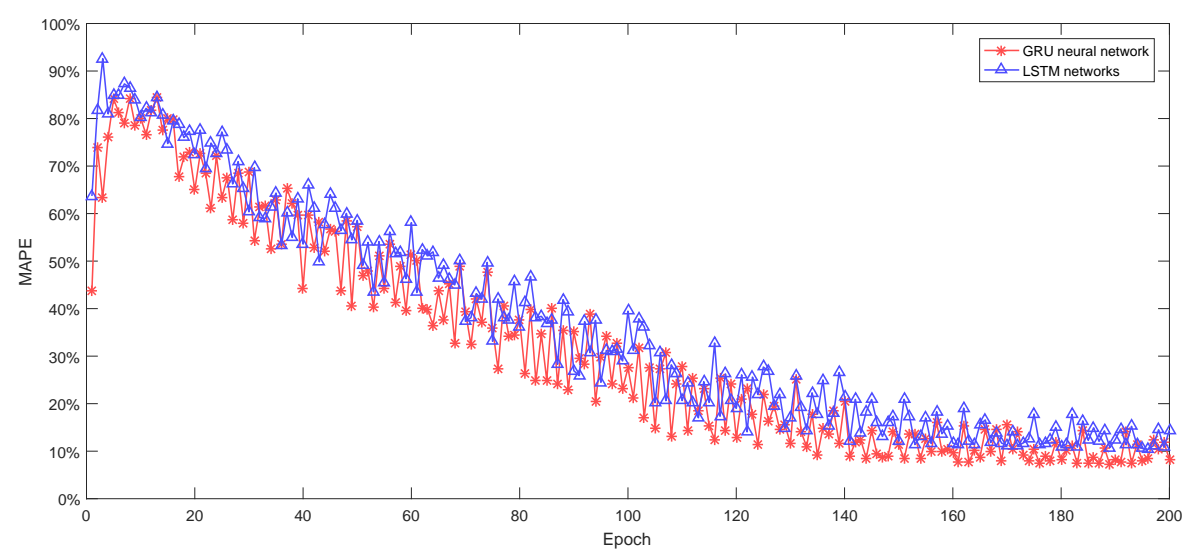

Figure 14. The MAPEs of network with LSTM and GRU layers in the same architecture with same samples during the training process.

We also performed the experiments to compare with current methods such as back-propagation neural networks (BPNNs) [7,8], stacked autoencoders (SAEs) [17], RNNs [24,25], and LSTM [29-31]. Their parameters and structures are set as described in Section 3.2. The compared average MAPEs of these methods, trained and tested with all samples described at the beginning of this subsection, are shown in Figure 15. The specific values of average and maximal MAPEs are shown in Table 10. Moreover, the results of nine customers are shown to validate the better performance of the proposed methods. The MAPEs for 30 November 2013 are shown in Table 11. It can be concluded that the proposed method results in smaller error in both average and maximal MAPEs. The proposed method performs better compared to the other current methods in most cases for short-term load forecasting in Wanjiang area.

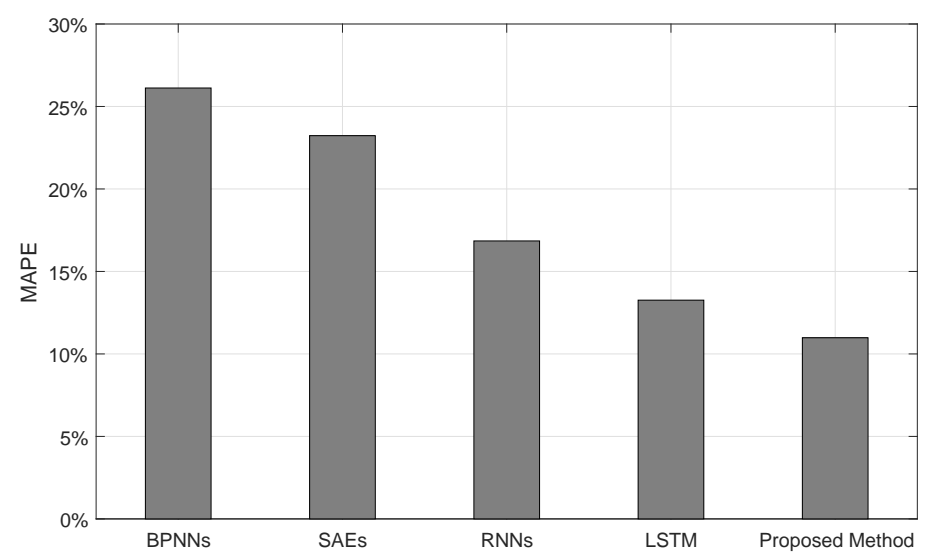

Figure 15. Compared average MAPEs trained and tested with all samples in three categories. 
Table 10. Average and maximal MAPEs of the proposed and current methods for short-term load forecasting.

\begin{tabular}{cccccc}
\hline Method & BPNNs & SAEs & RNNs & LSTM & Proposed Method \\
\hline Average MAPE & $26.12 \%$ & $23.23 \%$ & $16.85 \%$ & $13.26 \%$ & $\mathbf{1 0 . 9 8} \%$ \\
Maximal MAPE & $32.42 \%$ & $28.51 \%$ & $20.97 \%$ & $17.45 \%$ & $\mathbf{1 5 . 1 2} \%$ \\
\hline
\end{tabular}

Table 11. MAPEs of compared methods for nine customers' short-term load forecasting on 30 November 2013.

\begin{tabular}{cccccccc}
\hline Customer & Category & Feeder & BPNNs & SAEs & RNNs & LSTM & Proposed Method \\
\hline 37148000 & 1 & 2 & $26.96 \%$ & $23.28 \%$ & $16.56 \%$ & $13.09 \%$ & $\mathbf{1 0 . 8 0} \%$ \\
51690000 & 1 & 7 & $27.56 \%$ & $25.61 \%$ & $15.56 \%$ & $10.77 \%$ & $\mathbf{9 . 2 5} \%$ \\
37165000 & 1 & 7 & $28.55 \%$ & $24.81 \%$ & $13.35 \%$ & $14.55 \%$ & $\mathbf{1 1 . 1 2} \%$ \\
53990001 & 2 & 2 & $24.23 \%$ & $22.23 \%$ & $17.87 \%$ & $11.27 \%$ & $\mathbf{1 0 . 0 7} \%$ \\
54265001 & 2 & 3 & $26.33 \%$ & $27.56 \%$ & $17.23 \%$ & $12.22 \%$ & $\mathbf{1 1 . 9 1} \%$ \\
54265002 & 2 & 3 & $29.23 \%$ & $24.89 \%$ & $15.63 \%$ & $14.63 \%$ & $\mathbf{1 0 . 7 6} \%$ \\
31624001 & 3 & 35 & $30.45 \%$ & $22.56 \%$ & $19.93 \%$ & $\mathbf{1 2 . 2 9} \%$ & $13.56 \%$ \\
41661001 & 3 & 34 & $32.23 \%$ & $25.65 \%$ & $16.72 \%$ & $14.65 \%$ & $\mathbf{1 2 . 2 3} \%$ \\
76242001 & 3 & 33 & $25.36 \%$ & $23.22 \%$ & $14.66 \%$ & $13.27 \%$ & $\mathbf{9 . 9 8} \%$ \\
\hline
\end{tabular}

In detail, the forecasting load curves of Customers 37148000 and 53990001 on 30 November 2013 based on these methods are shown in Figures 16 and 17. We can observe the closest curve to the actual curve is the proposed method in the results of these experiments. Time information is important in short-term load forecasting which the BPNNs and SAEs cannot extract. Therefore, they get poorer performance in the experiments. The vanishing gradient problem limits the performance of RNNs because of the decreasing perception of nodes. The architecture is simpler and the parameters are fewer in GRU neural network compared to LSTM networks (Section 2.1). Therefore, the performances of GRU neural networks are better than the other current methods. In general, the availability and improvement of the proposed method are proven by the real-world experiments.

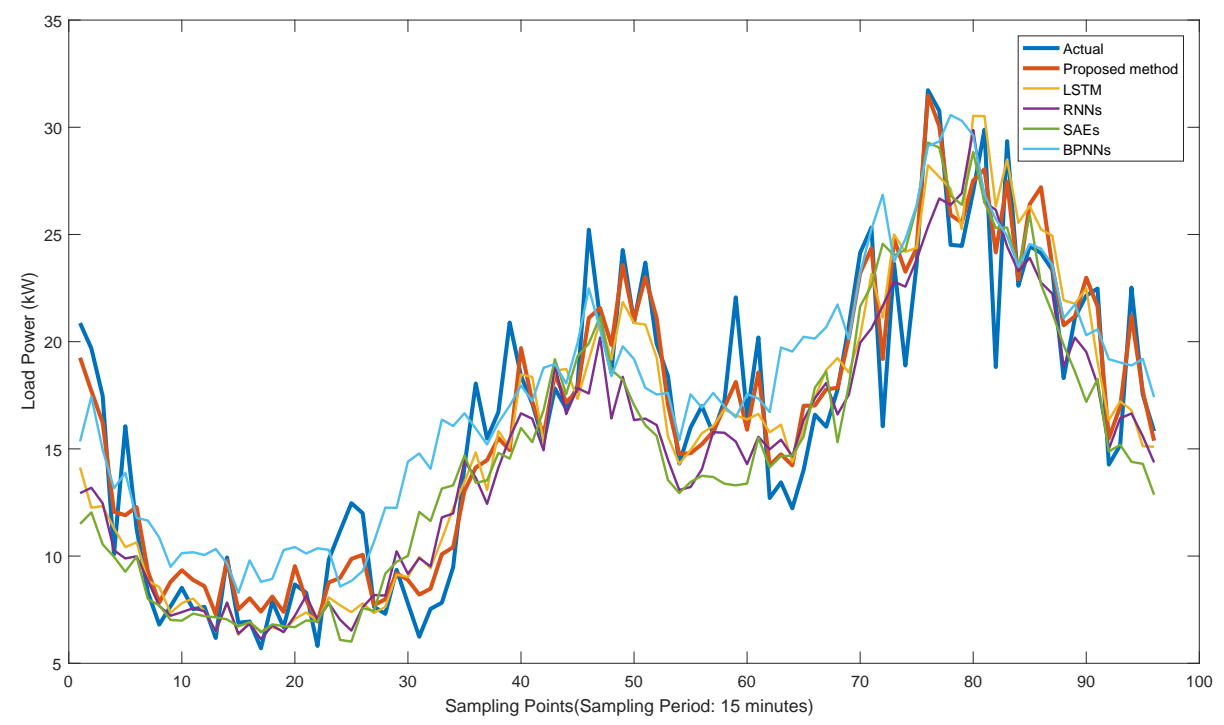

Figure 16. The load curves of Customer 37148000 based on the proposed method and the other current methods. 


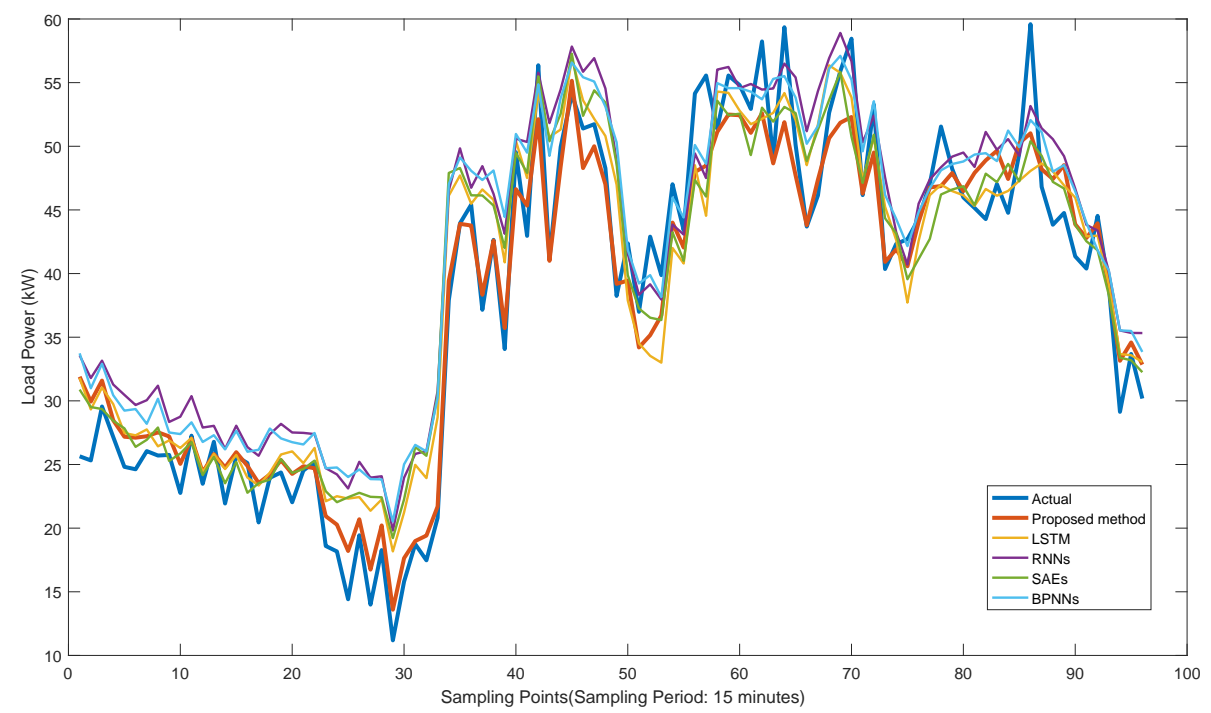

Figure 17. The load curves of Customer 53990001 based on the proposed method and the other current methods.

\section{Conclusions}

To increase the stability and economy of power grids, a method for short-term load forecasting with multi-source data using GRU neural networks is proposed in this paper, which focuses on individual customers. The proposed structure of the whole network is shown in Figure 5. The real-world load data of individual customers is recorded from Dongguan Power Supply Bureau of China Southern Power Grid in Guangdong Province, China. Before training, the customers with load data are clustered into three categories by K-means clustering algorithm to reduce the interference of different electricity use characteristics. Then, the environment factors are quantified and put into the input of the proposed networks for more information. The GRU units are introduced into the network for its simpler structure and faster convergence compared to LSTM blocks. The results in Figures 12 and 13 show that clustering and multi-source input can help to improve the performance of load forecasting. The average MAPE can be low as $10.98 \%$ for the proposed method, which outperforms the other current methods such as BPNNs, SAEs, RNNs and LSTM. The improvement is notable (Figures 15-17). In general, the availability and superiority of the proposed method are verified in this paper. In the future, combining with the technique of peak prediction could be a subject worth studying for load forecasting. Moreover, since the load forecasting for the customers in all power grid areas is a large-scale task, transfer learning and continuous learning will be considered based on the proposed framework for high-efficiency load forecasting.

Author Contributions: Y.W. and M.L. conceived the main idea; Z.B. and S.Z. collected the data and designed the experiments; Y.W., M.L. and Z.B. performed the experiments; and Y.W. wrote the paper.

Acknowledgments: This work is supported by the Zhejiang Provincial Natural Science Foundation of China under Grant LZ15F030001.

Conflicts of Interest: The authors declare no conflict of interest.

\section{References}

1. Espinoza, M.; Suykens, J.A.; Belmans, R.; De Moor, B. Electric load forecasting. IEEE Contr. Syst. Mag. 2007, 27, 43-57. [CrossRef]

2. Hong, T. Energy forecasting: Past, present, and future. Foresight Int. J. Appl. Forecast. 2014, 32, $43-48$.

3. Gross, G.; Galiana, F.D. Short-term load forecasting. Proc. IEEE 1987, 75, 1558-1573. [CrossRef] 
4. Hong, T.; Fan, S. Probabilistic electric load forecasting: A tutorial review. Int. J. Forecast. 2016, 32, 914-938. [CrossRef]

5. Taylor, J.W.; McSharry, P.E. Short-term load forecasting methods: An evaluation based on european data. IEEE Trans. Power Syst. 2008, 22, 2213-2219. [CrossRef]

6. Chen, H.; Wan, Q.; Zhang, B.; Li, F.; Wang, Y. Short-term load forecasting based on asymmetric ARCH models. In Proceedings of the Power and Energy Society General Meeting, Providence, RI, USA, 25-29 July 2010; pp. 1-6.

7. Quan, H.; Srinivasan, D.; Khosravi, A. Short-term load and wind power forecasting using neural network-based prediction intervals. IEEE Trans. Neural Netw. Learn. Syst. 2014, 22, 303-315. [CrossRef] [PubMed]

8. Ding, N.; Benoit, C.; Foggia, G.; Besanger, Y.; Wurtz, F. Neural network-based model design for short-term load forecast in distribution systems. IEEE Trans. Power Syst. 2016, 31, 72-81. [CrossRef]

9. Cevik, H.H.; CunkaS, M. Short-term load forecasting using fuzzy logic and ANFIS. Neural Comput. Appl. 2015, 26, 1355-1367. [CrossRef]

10. Lee, C.W.; Lin, B.Y. Applications of the chaotic quantum genetic algorithm with support vector regression in load forecasting. Energies 2017, 10, 1832. [CrossRef]

11. Hinton, G.E.; Salakhutdinov, R.R. Reducing the dimensionality of data with neural networks. Science 2006, 313, 504-507. [CrossRef] [PubMed]

12. Hinton, G.E.; Osindero, S.; Teh, Y.W. A fast learning algorithm for deep belief nets. Neural Comput. 2006, 18, 1527-1554. [CrossRef] [PubMed]

13. Chen, K.; Huang, C.; He, J. Fault detection, classification and location for transmission lines and distribution systems: A review on the methods. High Volt. 2016, 1, 25-33. [CrossRef]

14. Wang, Y.; Liu, M.; Bao, Z. Deep learning neural network for power system fault diagnosis. In Proceedings of the 35th Chinese Control Conference, Chengdu, China, 27-29 July 2016; pp. 6678-6683.

15. Ryu, S.; Noh, J.; Kim, H. Deep neural network based demand side short term load forecasting. Energies 2016, 10, 3. [CrossRef]

16. Qiu, X.; Ren, Y.; Suganthan, P.N.; Amaratunga, G.A. Empirical mode decomposition based ensemble deep learning for load demand time series forecasting. Appl. Soft Comput. 2017, 54, 246-255. [CrossRef]

17. Tong, C.; Li, J.; Lang, C.; Kong, F.; Niu, J.; Rodrigues, J.J. An efficient deep model for day-ahead electricity load forecasting with stacked denoising auto-encoders. J. Parallel Distrib. Comput. 2017, 1-7. [CrossRef]

18. Liao, Y.; Chen, S. A modular RNN-based method for continuous Mandarin speech recognition. IEEE Trans. Speech Aud. Proc. 2016, 9, 252-263. [CrossRef]

19. Nakashika, T.; Takiguchi, T.; Ariki, Y. Voice conversion using RNN pre-trained by recurrent temporal restricted Boltzmann machines. IEEE/ACM Trans. Aud. Speech Lang. Proc. 2015, 23, 580-587. [CrossRef]

20. Wu, H.; Prasad, S. Convolutional recurrent neural networks forhyperspectral data classification. Remote Sens. 2017, 9, 298. [CrossRef]

21. Zuo, H.; Fan, H.; Blasch, E.; Ling, H. Combining convolutional and recurrent neural networks for human skin detection. IEEE Signal Proc. Lett. 2017, 24, 289-293. [CrossRef]

22. Chien, J.T.; Ku, Y.C. Bayesian recurrent neural network for language modeling. IEEE Trans. Neural Netw. Learn. Syst. 2016, 27, 361-374. [CrossRef] [PubMed]

23. Ororbia, A.G., II; Mikolov, T.; Reitter, D. Learning simpler language models with the differential state framework. Neural Comput. 2017, 29, 3327-3352. [CrossRef] [PubMed]

24. Shi, H.; Xu, M.; Li, R. Deep learning for household load forecasting-A novel pooling deep RNN. IEEE Trans. Smart Grid 2017, 1-10. [CrossRef]

25. Wei, L.Y.; Tsai, C.H.; Chung, Y.C.; Liao, K.H.; Chueh, H.E.; Lin, J.S. A study of the hybrid recurrent neural network model for electricity loads forecasting. Int. J. Acad. Res. Account. Fin. Manag. Sci. 2017, 7, 21-29.

26. Hochreiter, S.; Schmidhuber, J. Long short-term memory. Neural Comput. 1997, 9, 1735-1780. [CrossRef] [PubMed]

27. Wei, D.; Wang, B.; Lin, G.; Liu, D.; Dong, Z.; Liu, H.; Liu, Y. Research on unstructured text data mining and fault classification based on RNN-LSTM with malfunction inspection report. Energies 2017, 10, 406. [CrossRef]

28. Zhang, S.; Wang, Y.; Liu, M.; Bao, Z. Data-based line trip fault prediction in power systems using LSTM networks and SVM. IEEE Access 2017, 6, 7675-7686. [CrossRef] 
29. Gensler, A.; Henze, J.; Sick, B.; Raabe, N. Deep Learning for solar power forecasting-An approach using AutoEncoder and LSTM Neural Networks. In Proceedings of the International Conference on Systems, Man, and Cybernetics, Budapest, Hungary, 9-12 October 2016; pp. 2858-2865.

30. Zheng, J.; Xu, C.; Zhang, Z.; Li, X. Electric load forecasting in smart grids using long-short-term-memory based recurrent neural network. In Proceedings of the 51st Annual Conference on Information Sciences and Systems, Baltimore, MD, USA, 22-24 March 2017; pp. 1-6.

31. Kong, W.; Dong, Z.Y.; Jia, Y.; Hill, D.J.; Xu, Y.; Zhang, Y. Short-term residential load forecasting based on LSTM recurrent neural network. IEEE Trans. Smart Grid 2017,1-11. [CrossRef]

32. Cho, K.; Van Merrienboer, B.; Gulcehre, C.; Bahdanau, D.; Bougares, F.; Schwenk, H.; Bengio, Y. Learning phrase representations using RNN encoder-decoder for statistical machine translation. arXiv 2014, arXiv:1406.1078.

33. Chung, J.; Gulcehre, C.; Cho, K.; Bengio, Y. Empirical evaluation of gated recurrent neural networks on sequence modeling. arXiv 2014, arXiv:1412.3555.

34. Lu, K.; Zhao, Y.; Wang, X.; Cheng, Y.; Pang, X.; Sun, W.; Jiang, Z.; Zhang, Y.; Xu, N.; Zhao, X. Short-term electricity load forecasting method based on multilayered self-normalizing GRU network. In Proceedings of the Conference on Energy Internet and Energy System Integration, Beijing, China, 26-28 November 2017; pp. 1-5.

35. Werbos, P.J. Backpropagation through time: What it does and how to do it. Proc. IEEE 1990, 78, 1550-1560. [CrossRef]

(C) 2018 by the authors. Licensee MDPI, Basel, Switzerland. This article is an open access article distributed under the terms and conditions of the Creative Commons Attribution (CC BY) license (http://creativecommons.org/licenses/by/4.0/). 